\title{
A review of the application of acoustic emission technique in engineering
}

\begin{abstract}
The use of acoustic emission (AE) technique for detecting and monitoring damages and the progress on damages in different structures is widely used and has earned a reputation as one of the most reliable and well-established technique in non-destructive testing (NDT). Acoustic Emission is a very efficient and effective technology used for fracture behavior and fatigue detection in metals, fiberglass, wood, composites, ceramics, concrete and plastics. It can also be used for detecting faults and pressure leaks in vessels, tanks, pipes, as well as for monitoring the progression of corrosion in welding. This paper reviews major research developments over the past few years in application of acoustic emission in numerous engineering fields, including manufacturing, civil, aerospace and material engineering.
\end{abstract}

Keywords: Application of AE; Acoustic emission; Non-destructive testing; NDT applications 